Analysis of thermal runaway in the ignition process

Dold, J W

1989

MIMS EPrint: 2007.86

Manchester Institute for Mathematical Sciences

School of Mathematics

The University of Manchester

\footnotetext{
Reports available from: http://eprints.maths.manchester.ac.uk/

And by contacting: The MIMS Secretary

School of Mathematics

The University of Manchester

Manchester, M13 9PL, UK
} 


\title{
ANALYSIS OF THERMAL RUNAWAY IN THE IGNITION PROCESS*
}

\author{
J. W. DOLD $\dagger$
}

\begin{abstract}
The evolution of a thermal runaway event is studied from the time a self-sustained temperature growth first sets in to the time deflagration flames begin to emerge. Proper modeling of the effect of conduction on the distribution of temperature growth reveals that it enhances the overall rate of release of chemical energy. It is shown that this contributes to the likelihood of substantial pressure increases being produced at some stage, and a criterion is identified for this to happen. In the absence of such pressure effects, the results are valid over a wide range of degrees of supercriticality, from marginal cases to cases in which conductive heat losses start off being very small indeed.
\end{abstract}

Key words. combustion, supercritical, unsteady, thermal runaway, ignition, bifurcation, ignition kernel, hot spot, Arrhenius kinetics

AMS(MOS) subject classifications. 35K 57, 41 A60, 76R99, 80A20

1. Introduction. Heat conduction can play an important role in the progress of thermal runaway and, in understanding this role, it is important to properly characterize its effects. Let us consider a nonuniform thermal evolution in a reacting combustible gas with large activation energy. If heat losses are sufficient then conduction can act to keep the temperature permanently in check. Situations of this nature have been extensively studied since the early work of Frank-Kamenetskii [1], [2] (see Zeldovich et al. [3]). However, if heat losses become inadequate (when conditions are said to be "supercritical") conduction fails to remove heat as fast as the reaction produces it; an unsteady growth in temperature becomes inevitable. Typically, a relatively hot kernel of self-heating reactants is formed [4]-[10] in which the exponentially nonlinear dependence of the rate of heat production on temperature leads to a vigorous and progressive acceleration of the peak temperature growth. The way in which any spatial variation in the temperature develops is primarily influenced by a combination of conduction and the nonlinear variation of reaction rate with temperature.

Since the hottest parts of a mixture tend to react and produce heat fastest, the temperature growth rate tends to be biased toward points with higher temperatures. Consequently, temperature differences tend to grow and the spatial region in which the reaction rate keeps pace with its maximum value tends to shrink as time proceeds. Just as heat conduction normally acts to spread out any such concentration of temperature, its effect in the evolving ignition kernel is to work against this focusing of temperature growth. Previous work has shown [8] that, once thermal runaway has begun, conduction acts to counter this focusing effect only by reducing the rate at which the peak temperature region becomes more and more localised. Along with this, conduction acts to progressively diminish its own importance as thermal runaway proceeds. A byproduct is that, in the core of the reacting region, the thermal growth is found to occupy more space than would be predicted without conduction; the overall rate of release of chemical energy is substantially increased. Thus while conduction may normally be thought of as working to inhibit thermal runaway, it also brings about an increase in the power released by an evolving ignition kernel.

* Received by the editors June 29, 1987; accepted for publication (in revised form) February 15, 1988. This work was carried out with the support of the Science and Engineering Research Council, as part of its initiative in funding nonlinear mathematical research.

† School of Mathematics, University of Bristol, University Walk, Bristol BS8 1TW, United Kingdom. 
Until recently, an alternative picture was proposed for describing thermal runaway [5]-[9], in which the relative effect of conduction to that of the reaction would not diminish as time proceeds (see footnote 1). Attempts to numerically obtain such solutions failed [8], [9] and rigorous analysis has now proven that these solutions do not exist in a wide range of situations [11]. This is interesting because these conjectured solutions take the form that might be expected from a self-similarity analysis of the governing equations based on dimensional arguments [6]. The true picture is brought about by a fully nonlinear interaction between conductive effects and the growth of the reaction rate with temperature [8].

In this paper a complete asymptotic solution is presented for thermal runaway, from its early stage (already presented in [8]) to a stage when deflagration flames can be described. This might be said to mark the completion of an ignition process; an unsteady transition has taken place from a relatively cold, slowly reacting state to a state in which hot flames begin to propagate.

It is noted that expansion velocities reach overall maximum values in the final stages of thermal runaway. It is conjectured that this velocity could form a large enough fraction of the speed of sound for the modeling, which is based on assuming low Mach-number flows, to break down. Under these conditions it may be possible to observe a transition from near-constant pressure behaviour to near-constant density with significant increases in absolute pressure [12]. This is more likely to happen in highly supercritical cases for which diffusion plays a small part right from the start of thermal runaway.

\section{Model.}

2.1. Low Mach number equations. In order to capture the most essential properties of a combustible gaseous mixture in the simplest mathematical model, we assume that a single exothermic one-step chemical reaction, $v_{F} F+v_{X} X \rightarrow v_{P} P$, occurs between $v_{F}$ molecules of a "fuel" $F$ and $v_{X}$ molecules of an "oxidant" $X$ to produce $v_{P}$ molecules of the product $P$. It is also simplest to consider dilute gaseous mixtures (consisting mostly of some chemically neutral component) so that, for instance, the mean molecular weight $W$ and specific heat at constant pressure $C_{p}^{\#}$ can realistically be taken to stay more or less constant. As soon as a suitable set of fixed reference values for the various gas properties is identified, the system of equations governing the progress of the reaction can be expressed in a nondimensional form. This procedure is described in the following paragraphs (where the superscript \# is used to indicate the dimensional value of a quantity).

The symbols $T_{c}^{\#}, \mathscr{P}_{c}^{\#}, \lambda_{c}^{\#}$, and $\eta_{c}^{\#}$ will be used to denote appropriately chosen typical values of the absolute temperature, absolute pressure, thermal conductivity, and coefficient of dynamic viscosity, respectively. The thermal equation of state for an ideal gas then identifies a typical value for the density, namely $\rho_{c}^{\#}=\mathscr{P}_{c}^{\#} W /\left(R^{\#} T_{c}^{\#}\right)$ where $R^{\#}$ is the universal gas constant. A typical value for the thermal diffusivity is given by $k_{c}^{\#}=\lambda_{c}^{\#} /\left(\rho_{c}^{\#} C_{p}^{\#}\right)$. If we assume that Fick's law is adequate for describing the diffusion of mass, then there must be a single typical value $\mathscr{D}_{c}^{\#}$ for the diffusion coefficient. The Prandtl and Lewis numbers, which we take to be constant and comparable with unity in value for most gases, are then given by $\operatorname{Pr}=\eta_{c}^{\#} /\left(\rho_{c}^{\#} k_{c}^{\#}\right)$ and (in its classical definition) $\mathrm{Le}=\mathscr{D}_{c}^{\#} / k_{c}^{\#}$, respectively.

If $t_{c}^{\#}$ is selected to be a measure of the time it takes for ignition to occur, then it becomes possible to define a typical thermal diffusion velocity $u_{c}^{\#}=\left(k_{c}^{\#} / t_{c}^{\#}\right)^{1 / 2}$ and a typical diffusion length $r_{c}^{\#}=u_{c}^{\#} t_{c}^{\#}=\left(k_{c}^{\#} t_{c}^{\#}\right)^{1 / 2}$. In situations where diffusive phenomena (including thermal conduction) are anticipated to be the most significant processes 
governing the transport of heat and matter, $u_{c}^{\#}$ and $r_{c}^{\#}$ should be suitable reference values against which to measure gas flow velocities and distances.

To be realistic, it is important to note that because relatively few molecular collisions actually result in a chemical change, the appropriate timescale for the chemistry is inevitably much longer than the mean molecular collision time. Thus in any chemical reaction, the ratio of the time taken for the rate of reaction to evolve and change by an order-one amount, to the mean molecular collision time, is always a large number. This ratio is sometimes known [20] as the von Kármán number Ká, an appropriate typical value that can be identified as $\mathrm{Ka}_{c}=t_{c}^{\#} a_{c}^{\# 2} / k_{c}^{\#}$, after noting that $k_{c}^{\#} / a_{c}^{\# 2}$ is a measure of the typical mean molecular collision time where $a_{c}^{\#}$ is a typical sound speed. A suitable expression for $a_{c}^{\#}$ is $a_{c}^{\#}=\left(\gamma \mathscr{P}_{c}^{\#} / \rho_{c}^{\#}\right)^{1 / 2}=$ $\left((\gamma-1) C_{p}^{\#} T_{c}^{\#}\right)^{1 / 2}$, where $\gamma$ is the ratio of principal specific heats (most usefully calculated under conditions of fixed chemical composition). The resulting typical diffusional Mach number $M$, defined as the ratio $M=u_{c}^{\#} / a_{c}^{\#}=K_{a}^{-1 / 2}$, is then an important parameter in combustible systems primarily because it is invariably small.

In situations where diffusion is the main transport mechanism, actual gas velocities are similar in magnitude to $u_{c}^{\#}$ so that the gas-flow Mach number is also of the order of $M$. Under such circumstances, local pressures do not, in general, change very dramatically from the average system value; variations from $\mathscr{P}_{c}^{\#}$ are typically of the order of $\rho_{c}^{\#} u_{c}^{\# 2}$ or $\gamma M^{2} \mathscr{P}_{c}^{\#}$, although we should perhaps recognize that if acoustic, or gas-dynamical, pressure changes were to play a significant part then these would best be measured in units of $\rho_{c}^{\#} a_{c}^{\#} u_{c}^{\#}$ or $\gamma M \mathscr{P}_{c}^{\#}$ on a fast timescale of order $r_{c}^{\#} / a_{c}^{\#}$ or $M t_{c}^{\#}$.

The rate of progress $\mathscr{R}^{\#}$ of the chemical reaction can be taken to satisfy Arrhenius kinetics:

$$
\mathscr{R}^{\#}=\left(\rho^{\#} c_{F}\right)^{v_{F}}\left(\rho^{\#} c_{X}\right)^{v_{x}} F^{\#}\left(T^{\#}\right) e^{-E^{*} /\left(R^{*} T^{*}\right)}
$$

in which $E^{\#}$ is the molar activation energy of the reaction, and for the chemical species $\kappa, c_{\kappa}$ denotes the mass fraction of the species at a point; the function $F^{\#}\left(T^{\#}\right)$ is a pre-exponential frequency factor with the dimensions of density to the power $1-v_{F}-v_{X}$ divided by time. It is not necessary to actually specify $F^{\#}\left(T^{\#}\right)$ for the analysis below to remain valid. In units of mass per unit volume per unit time, the rate of production of a chemical species is given by $-v_{\kappa} W_{\kappa} \mathscr{R}^{\#}$ (when $\kappa$ represents either $F$ or $X$ and $W_{\kappa}$ is the molecular weight of the species $\kappa$ ) or by $v_{P} W_{P} \mathscr{R}^{\#}$ for the product species $P$.

If we define the dimensionless activation energy as $\theta=E^{\#} /\left(R^{\#} T_{c}^{\#}\right)$ it transpires that a useful form for the time $t_{c}^{\#}$ is

$$
t_{c}^{\#}=\rho_{c}^{\#} C_{p}^{\#} T_{c}^{\#} e^{\theta} /\left[\theta v_{P} W_{P} Q^{\#}\left(\rho_{c}^{\#} \mathscr{C}_{F}\right)^{v_{F}}\left(\rho_{c}^{\#} \mathscr{C}_{X}\right)^{v_{x}} F^{\#}\left(T_{c}^{\#}\right)\right]
$$

in which the constants $\mathscr{C}_{F}$ and $\mathscr{C}_{X}$ are chosen to be of the same order of magnitude as the values of $c_{F}$ and $c_{X}$ before substantial chemical changes occur, and $Q^{\#}$ is the chemical energy per unit mass released by the reaction. The nondimensional activation energy $\theta$ is generally found to have a large value and, in common with much successful combustion modeling of single step reactions, this property will be exploited here to solve the governing equations using asymptotic techniques.

By continuing to use the subscript $\kappa$ to denote either $F$ or $X$, the equations that describe the system can now be written in the following dimensionless forms [13], [14]: Species:

$$
\rho^{\prime}\left(y_{\kappa t}^{\prime}+\underline{u}^{\prime} \cdot \nabla y_{\kappa}^{\prime}-\operatorname{Le} \nabla \cdot\left(\rho^{\prime} \mathscr{D}^{\prime} \nabla y_{\kappa}^{\prime}\right)=-\mathscr{R}^{\prime} /\left(\theta \zeta_{k}\right)\right.
$$

Energy:

(2.4) $\rho^{\prime}\left(T_{t}^{\prime}+\underline{u}^{\prime} \cdot \nabla T^{\prime}\right)-\nabla \cdot\left(\lambda^{\prime} \nabla T^{\prime}\right)=\mathscr{R}^{\prime} / \theta+(\gamma-1) M^{2}\left\{p_{t}^{\prime}+\underline{u}^{\prime} \cdot \nabla p^{\prime}-\operatorname{Pr} \eta^{\prime}\left[S^{\prime}\right]:\left[\nabla \underline{u}^{\prime}\right]\right\} ;$ 
Continuity:

$$
\rho_{t}^{\prime}+\nabla \cdot\left(\rho^{\prime} \underline{u}^{\prime}\right)=0
$$

Momentum:

$$
\rho^{\prime}\left[\underline{u}_{t}^{\prime}+\left(\underline{u}^{\prime} \cdot \nabla\right) \underline{u}^{\prime}\right]+\nabla p^{\prime}=\operatorname{Pr} \nabla \cdot\left(\eta^{\prime}\left[S^{\prime}\right]\right) ;
$$

Gas Equation of State:

$$
\rho^{\prime} T^{\prime}=1+\gamma M^{2} p^{\prime}
$$

where

$$
\begin{gathered}
\mathscr{R}^{\prime}=\left(\rho^{\prime} y_{F}^{\prime}\right)^{v_{F}}\left(\rho^{\prime} y_{X}^{\prime}\right)^{v_{x}} F\left(T^{\prime}\right) e^{\theta\left(1-1 / T^{\prime}\right)} \\
{\left[S^{\prime}\right]=\left[\nabla \underline{u}^{\prime}\right]+\left[\nabla \underline{u}^{\prime}\right]^{T}-\frac{2}{3}\left(\nabla \cdot \underline{u}^{\prime}\right)[I]}
\end{gathered}
$$

(in which $[I]$ is the unit tensor).

The nondimensional values of the coordinates and various gas properties appearing in these equations are defined such that $\underline{r}=\underline{r}^{\#} / r_{c}^{\#}, t=t^{*} / t_{c}^{\#}, \underline{u}^{\prime}=\underline{u}^{\#} / u_{c}^{\#}, T^{\prime}=T^{\#} / T_{c}^{\#}$, $\rho^{\prime}=\rho^{\#} / \rho_{c}^{\#}, y_{\kappa}^{\prime}=c_{\kappa} / \mathscr{C}_{\kappa}, \quad \zeta_{\kappa}=v_{P} W_{P} Q^{\#} \mathscr{C}_{\kappa} /\left(v_{\kappa} W_{k} C_{p}^{\#} T_{c}^{\#}\right), \lambda^{\prime}=\lambda^{\#} / \lambda_{c}^{\#}, \mathscr{D}^{\prime}=\mathscr{D}^{\#} / \mathscr{D}_{c}^{\#}$, $\eta^{\prime}=\eta^{\#} / \eta_{c}^{\#}, p^{\prime}=\left(\mathscr{P}^{\#}-\mathscr{P}_{c}^{\#}\right) /\left(\rho_{c}^{\#} u_{c}^{\# 2}\right)$, and $F\left(T^{\prime}\right)=F^{\#}\left(T^{\prime} T_{c}^{\#}\right) / F^{\#}\left(T_{c}^{\#}\right)$, with $\nabla$ representing the gradient operator in terms of the dimensionless coordinate $\underline{r}$. The constants $\zeta_{\kappa}$ are nondimensional measures of the amount of chemical heat release (or temperature rise), which is potentially available in the concentrations of the reactants.

A useful alternative form of the continuity equation can be derived by first using the thermal equation of state (2.7) to eliminate $\rho_{t}^{\prime}+\underline{u}^{\prime} \cdot \nabla \rho^{\prime}$, and then using the resulting equation to eliminate $T_{t}^{\prime}$ from the energy equation (2.4). This gives

$$
M^{2} p_{t}^{\prime}+\nabla \cdot\left(\rho^{\prime} T^{\prime} \underline{u}^{\prime}-\lambda^{\prime} \nabla T^{\prime}\right)=\mathscr{R}^{\prime} / \theta+(\gamma-1) M^{2}\left\{\underline{u}^{\prime} \cdot \nabla p^{\prime}+\operatorname{Pr} \eta^{\prime}\left[S^{\prime}\right]:\left[\nabla \underline{u}^{\prime}\right]\right\} .
$$

When we use these equations, the fact that the diffusional Mach number $M$ is small can now be seen to have some useful consequences for the modeling. If it is formally assumed that all of the dependent variables (represented by $f^{\prime}$ ) behave in the asymptotic manner $\mathrm{f}^{\prime}(r, t ; M)=\mathbf{f}(r, t)+O\left(M^{2}\right)$ as $M$ tends to zero, then the asymptotic forms (unprimed) of the dependent variables will satisfy equations (2.3)-(2.10) with the primes and the terms containing $M$ omitted.

The asymptotic form of (2.10), namely

$$
\nabla \cdot(\underline{u}-\lambda \nabla T)=\mathscr{R} / \theta,
$$

is of particular interest since it establishes a relationship between the divergence of the flow and, in essence, thermal expansion effects associated with either thermal conduction or chemical heating. The momentum equation (2.6)-with the primes omitted-then helps to determine the weak pressure gradients, $\nabla p$, which are required to drive any velocity changes.

A limitation in the asymptotic modeling is also revealed by equations (2.6) and (2.11) as soon as it is noticed that these equations are unable to describe acoustic interactions between pressure and velocity. As indicated earlier, different pressure and timescales could be used to admit a description of an acoustic field, but this aspect is not of particular importance here. Attention is concentrated specifically on the wide class of cases in which acoustic interactions remain of minor importance during the various stages of ignition. A distinguished limit is discussed later (see (6.26)) under which gasdynamic interactions would become significant; some analysis of the 
consequent behaviour, as well as the wave field produced by thermal expansion in an ignition kernel, is presented elsewhere [12].

Under conditions of symmetry and nonrotation, (2.11) takes the form $r^{-n}\left[r^{n}(u-\right.$ $\left.\left.\lambda T_{r}\right)\right]_{r}=\mathscr{R} / \theta$ where $u$ and $r$ are now the radial components of velocity and position, respectively. The number $n$ depends solely on the geometry of the situation being considered, taking the values: zero for variation in only one Cartesian dimension, with planar symmetry about $r=0$; one for cylindrical symmetry; and two for spherical symmetry. Because $u$ must now satisfy the condition $u(0, t) \equiv 0$, the equation can be integrated to give

$$
u=\lambda T_{r}+\frac{v}{\theta}
$$

with

$$
v=r^{-n} \int_{0}^{r} r^{n} \mathscr{R} \partial r
$$

Thus the quantity $v / \theta$ represents the component of velocity, which is due entirely to thermal expansion brought about by the chemical heating term $\mathscr{R} / \theta$. Equation (2.12) shows that velocity consists solely of thermal conductive and chemical heating induced expansion effects.

Using this result to eliminate $u$ from the convective terms of the appropriate symmetric forms of the species and energy equations, we obtain

$$
y_{\kappa t}+\left[\frac{v}{\theta}+\lambda T_{r}-\operatorname{Le} T(\rho \mathscr{D})_{r}\right] y_{\kappa r}-\operatorname{Le} \mathscr{D} r^{-n}\left(r^{n} y_{\kappa r}\right)_{r}=-T \frac{\mathscr{R}}{\theta \zeta_{\kappa}}
$$

and

$$
T_{t}+\left[\frac{v}{\theta}+\lambda T_{r}-T \lambda_{r}\right] T_{r}-T \lambda r^{-n}\left(r^{n} T_{r}\right)_{r}=T \frac{\mathscr{R}}{\theta} .
$$

These equations show that a considerable simplification will result if $\lambda, \rho \mathscr{D}$, and Le are modeled (not unrealistically) such that $\lambda=\rho \mathscr{D}=T$ and Le $=1$. Further simplification follows through identifying the conserved scalars (quantities not consumed by the reaction),

$$
Y_{\kappa}=y_{\kappa}+(T-1) / \zeta_{\kappa} \text {. }
$$

Sufficient equations with which to describe the behavior of $Y_{F}, Y_{X}$, and $T$ can now be written in as follows:

$$
Y_{\kappa t}+\frac{v}{\theta} Y_{\kappa r}=T^{2}\left(Y_{\kappa r r}+\frac{n}{r} Y_{\kappa r}\right)
$$

and

$$
T_{t}+\frac{v}{\theta} T_{r}=\frac{T \mathscr{R}}{\theta}+T^{2}\left(T_{r r}+\frac{n}{r} T_{r}\right)
$$

where

$$
\mathscr{R}=\left[\frac{1}{T}\left(Y_{F}-\frac{T-1}{\zeta_{F}}\right)\right]^{v_{F}}\left[\frac{1}{T}\left(Y_{X}-\frac{T-1}{\zeta_{X}}\right)\right]^{v_{X}} F(T) e^{\theta(1-1 / T)} .
$$

A combination of the asymptotic forms of the continuity, energy, and momentum 
equations can also be used to derive the following form of the momentum equation:

$$
T p_{r}+\frac{5}{2} n u T T_{r} / r+\theta^{-1}\left(v_{t}+u v_{r}\right)=\left(\frac{4}{3} \operatorname{Pr}-1\right) T\left\{\eta\left[r^{-n}\left(r^{n} u\right)_{r}\right]_{r}+\eta_{r}\left(u_{r}-\frac{1}{2} n u / r\right)\right\}
$$

in which an obvious further simplification follows if the Prandtl number takes the value $\frac{3}{4}$. With $(2.12)$ this serves to uniquely determine the velocity $u$ and pressure gradient $p_{r}$ in terms of the species and temperature solutions of equations (2.17)-(2.19).

2.2. Conditions for ignition. Studies have shown [1]-[10] that when equations of this type are used, a rapid thermal runaway through self-heating will always occur provided sufficient fuel and oxidant are available at some initial instant, and heat losses are small enough. The result can only be the generation of some kind of vigorous combustion. Such an ignition process takes place in a small region located about some position $r=\hat{r}(\theta)$ in a short space of time close to some time $t=\hat{t}(\theta)$. Selecting the nondimensionalization such that $T$ is initially within order $\theta^{-1}$ of unity, we have that $\hat{r}$ and $\hat{t}$ are typically found to adopt order-one values on the scalings of space and time shown in (2.3)-(2.20). This is in accordance with $t_{c}^{\#}$ being a measure of the dimensional induction time. First, we will discuss some necessary conditions associated with the occurrence of ignition.

Since we are mainly concerned with analysing a very localized and very rapid thermal runaway process, it is sufficient for the purposes of this paper to assume that $Y_{F}$ and $Y_{X}$ are known to vary as follows:

$$
Y_{\kappa}(r, t)=\hat{Y}_{\kappa}+O(r-\hat{r})+O(t-\hat{t}) .
$$

In a uniformly premixed system, for instance, $Y_{F}$ and $Y_{X}$ would satisfy this condition exactly. Otherwise, derivatives of $Y_{F}$ and $Y_{X}$ with respect to $r$ and $t$ are generally of order one at the ignition event [10]. Hence changes in these quantities can be ignored when variations in $R$ and $t$ from the values $\hat{r}$ and $\hat{t}$ are small, as is the case in the relatively small ignition region.

The thermal runaway process is characterized by a localized self-heating, which progressively and dramatically accelerates under the exponential influence of the Arrhenius reaction-rate law. The growth of any such temperature peak only slows down again when $y_{F}$ or $y_{X}$ approaches zero. From (2.16) and (2.19) it can be seen that this occurs when $T=T_{b}(r, t)-O\left(\theta^{-1}\right)$ where

$$
T_{b}= \begin{cases}1+\zeta_{F} Y_{F}, & \zeta_{F} Y_{F} \leqq \zeta_{X} Y_{X}, \\ 1+\zeta_{X} Y_{X}, & \zeta_{X} Y_{X} \leqq \zeta_{F} Y_{F} .\end{cases}
$$

When $\hat{r}$ and $\hat{\mathbf{t}}$ characterize the first attainment of this fastest stage of the reaction, the condition that ignition occurs leads to the requirement that

$$
T(\hat{r}, \hat{t})=1+\zeta_{L} \hat{Y}_{L}-O\left(\theta^{-1}\right)
$$

with $T(r, \hat{t})$ bounded above by its maximum value $T(\hat{r}, \hat{t})$ at $r=\hat{r}$ (by definition). The subscript $L$ denotes the locally lean reactant at the ignition event. More generally, if $R$ denotes the rich reactant at a time and place then $L$ and $R$ are defined such that $\zeta_{L} Y_{L}(r, t) \leq \zeta_{R} Y_{R}(r, t)$. In the case of stoichiometry (that is, $\zeta_{F} Y_{F}=\zeta_{X} Y_{X}$ ) the choice is arbitrary.

We should remark here that the discussion of the model for thermal runaway has not yet included any specification of boundary and initial conditions that may give rise to it. A great deal of analysis has been carried out characterizing steady or quasi-steady solutions associated with the equations above, under a variety of conditions [3]. These solutions generally show bifurcations that indicate, for example, that 
no steady slowly-reacting solution can exist in ranges of parameters (associated with the boundary conditions) beyond critical values where the steady solutions experience turning points. Under such "supercritical" conditions, temperatures cannot indefinitely remain near any relatively cold initial values, and ignition must ensue. Some numerical studies [4]-[10] have been carried out into the early development of thermal runaway from a variety of initial and boundary arrangements.

In general, the ignition position and time $\hat{r}$ and $\hat{t}$, the degree of symmetry of the ignition kernel (depending on $n$ and $\hat{r}$ ) as well, of course, as the very existence of thermal runaway, depend intimately on the conditions leading to ignition. However, once thermal runaway has begun and these features are known, the local structure and behavior of the ignition process can, to a large extent, be studied in isolation; broad classes of conditions, ranging from initially uniform mixtures in fixed temperature containers to spark ignition and thermal runaway in nonpremixed systems, can be expected to lead to essentially the same structure of ignition kernel. It is not the intention of this paper to restrict attention to any specific class of initial and boundary data, and an investigation of all such classes is beyond its scope. It is intended rather to maintain generality by being primarily concerned with the local details of the ignition process itself.

It has already been demonstrated [8] that the early structure of an ignition kernel is remarkably independent both of the conditions that lead to its formation, and its geometrical shape (whether it is symmetric about a point, line, or plane). However, one major characteristic that does emerge is that conductive effects diminish in importance, relative to the reaction rate, as thermal runaway proceeds. In this respect, a strongly supercritical ignition kernel that starts off only weakly influenced by conduction is very much like a more fully developed ignition kernel for which conduction has become less significant. As presented in the next section, this adds one more feature that needs to be included as a condition for studying the full thermal runaway process, but olierwise it is not really necessary to consider the outer problem from which thermal runaway arises; with a great deal of generality, the evolution of the ignition kernel can be studied in isolation from the detailed conditions that produce it.

3. Early temperature growth. With $T$ initially close to unity in value, the early temperature behaviour can be modeled asymptotically, for large values of $\theta$, by defining $\phi$ such that

$$
T(r, t ; \theta)=1+\theta^{-1} \phi(r, t)+O\left(\theta^{-2}\right) .
$$

Equation (2.18) then becomes

$$
\phi_{t}=\phi_{r r}+\frac{n}{r} \phi_{r}+\Lambda e^{\phi}
$$

where $\Lambda(r, t)=Y_{F}^{v_{F}} Y_{X}^{v_{X}}$, and condition (2.23) requires that

$$
\phi(\hat{r}, \hat{t})=\infty
$$

Although (3.2) becomes invalid when $\phi$ grows to the order of $\theta$, the way in which $\phi$ approaches the singularity (3.3) provides a first step in determining the full development of the thermal runaway. Without any spatial variation [15] the solution for $\phi$ is very simply, $\phi=-\ln [\hat{\Lambda}(\hat{t}-t)]$ where $\hat{\Lambda}=\Lambda(\hat{r}, \hat{t})$.

If, as would usually be the case, the variation of $\phi$ is not spatially uniform then it has been shown [8] that the singular growth of $\phi$ satisfies the well-defined asymptotic 
form $^{1}$ as $t \rightarrow \hat{t}$ :

$$
\begin{aligned}
\phi= & -\ln [\hat{\Lambda}(\hat{t}-t)]-\ln \left(1+\frac{\rho^{2}}{4}\right)-\frac{\ln \xi}{\xi} \frac{5+\hat{n}}{2} \frac{\rho^{2} / 4}{1+\rho^{2} / 4} \\
& +\xi^{-1}\left[\frac{1+\hat{n}}{2}-\frac{\rho^{2} / 4}{1+\rho^{2} / 4} \ln \left(1+\frac{\rho^{2}}{4}\right)\right]+O\left(\frac{\ln ^{2} \xi}{\xi^{2}}\right)
\end{aligned}
$$

where

$$
(\hat{t}-t) \hat{\Lambda}=e^{\alpha-\xi}
$$

The constant $\alpha$ depends on the initial or boundary conditions that give rise to thermal runaway. Broadly speaking, $\alpha$ increases with the degree of supercriticality of these conditions and can vary from order one to very large values [8]. The variable grouping $\rho$, which is defined such that

$$
r-\hat{r}=\mu \rho: \quad \mu^{2}=\xi \cdot(\hat{t}-t),
$$

characterizes the spatial concentration with time of the solution (3.4) for the peak temperature growth. Because $\xi$ increases as $\alpha$ increases, it can be seen that the spatial size of the ignition region at a given peak temperature is larger for larger values of $\alpha$. Correspondingly, the effect of conduction in (3.2) is smaller in relation to the effect of the reaction rate term. The geometrical factor $\hat{n}$, for the ignition region, is defined as $n$ if $\hat{r}=0$, or 0 if $\hat{r}>0$.

This result is remarkable in that, to first order as $\xi \rightarrow \infty$ with $\alpha$ finite, the growth and spatial variation of $\phi$ are independent of both the geometry $\hat{n}$ of the ignition region and the conditions leading to thermal runaway (on which $\alpha$ depends). It is shown in [8] that this behavior, and in particular the form of the grouping $\rho$, arise from both the diffusive nature and nonlinearity of (3.2). However $\alpha$ can have large values, comparable in magnitude to (or even much larger than) $\theta$, which typically lies between about 10 and 50 in value. Under these circumstances, $\alpha$ would in fact substantially affect the first-order spatial variation of the temperature $T$ unless $\xi-\alpha$, and hence $\phi$, could become unrealistically large.

It is worth noting at this point that, as an asymptotic solution to (3.2), the solution (3.4) remains valid as $t \rightarrow \hat{t}$ however large $\alpha$ may be. Indeed, since values of $\xi$ are greater for larger values of $\alpha$, the neglected terms actually become smaller, tending to make the expansion more accurate as $\alpha$ is increased. In the limit as $\alpha \rightarrow \infty$ for finite values of $r$ and $\hat{t}-t,(3.5)$ and (3.6) show that $\rho \rightarrow 0$ and $\xi \rightarrow \infty$. The solution thus converges to the exact solution without spatial variation, already described above. There is also no breakdown in the asymptotic structure (3.1) implied by allowing $\alpha$ to be possibly much greater than $\theta$.

\footnotetext{
${ }^{1}$ Until recently it was postulated [5], [6] that the growth of $\phi$ could adopt the alternative form of solution $\phi \sim-\ln (\hat{t}-t)+\phi_{1}\left[(r-\hat{r}) /(\hat{t}-t)^{1 / 2}\right]$, as might superficially be expected from dimensional similarity arguments associated with (3.2). It turns out [8], [11] that no physically meaningful solutions exist that take this asymptotic form. However, Buckmaster [16] has suggested that the differences between this alternative form and the correct solution for temperature growth are only of mathematical interest and make no qualitative difference to the physical results. In fact, as will be seen in $\S 6$, the predicted size of the ignition kernel can be an order of magnitude larger and correspondingly more energetic; this could make a substantial difference to gasdynamic aspects, possibly introducing significant pressure effects in the ignition process [12]. Also, the nature of the way in which deflagration flames finally emerge, as described in $\S 7$, is qualitatively very different.
} 
It will be seen in the next section that such large values of $\alpha$ are easily allowed for in developing an asymptotic solution of wide validity. The only limitation on the size of $\alpha$ arises through the possibility of the low Mach number modeling breaking down. The way in which this can happen and its implication for the allowable size of $\alpha$ are discussed in $\S 6$.

While describing the growth of the temperature peak, the asymptotic form (3.4) is not valid for large values of $\rho$ (namely when $\ln \rho^{2}$ becomes of the order of $\ln \xi$ ). Because of the definition of $\rho$, this restricts the range of values of $r$ for which the solution is valid to a "shrinking" neighborhood of $\hat{r}$ as $t$ approaches $\hat{t}$. The asymptotic solution [8],

$$
\phi=\chi-\alpha-\ln (1-\lambda)-\frac{\ln \chi}{\chi} \frac{5+\hat{n}}{2} \frac{1}{1-\lambda}+\chi^{-1}\left[\frac{1+\hat{n}}{2}-\frac{1}{1-\lambda} \ln (1-\lambda)\right]+O\left(\frac{\ln ^{2} \chi}{\chi^{2}}\right)
$$

in which $\chi$ and $\lambda$ are defined such that $(r-\hat{r})^{2} \hat{\Lambda} / 4=\chi e^{\alpha-\chi}$ and $(t-\hat{t}) \hat{\Lambda}=\lambda e^{\alpha-\chi}$, complements the solution (3.4) by describing the temperature in regions which, at some stage, are left "outside" the shrinking peak-temperature region as $t \rightarrow \hat{t}$. As mentioned earlier, (3.2) and both of these solutions become invalid when $\phi$ becomes of order $\theta$. At this stage $T-1$ is no longer small and the consumption of reactants, which is not represented in (3.2), becomes significant.

4. Substantial temperature growth. In modeling homogeneous thermal runaway, Kassoy [15] made use of the time-stretching transformation

$$
(\hat{t}-t) \hat{\Lambda}=e^{-\theta s+g(s ; \theta)} .
$$

This is equally useful in the present context where $\mu^{2}$ in the definition (3.6) of $\rho$ now becomes $\mu^{2}=(\alpha+\theta s-g)(\hat{t}-t)$. Still following Kassoy, it is useful to define a temperature-related variable $\eta$ such that

$$
\eta=\theta(1-1 / T-s) \text {. }
$$

The degree of freedom afforded by the choice of the function $g(s ; \theta)$ in (4.1) can now be exploited to normalize $\eta$ in some convenient way. Perhaps the simplest choice is to select the value zero for $\eta$ at $\rho=0$ :

$$
\eta(0, s ; \theta) \equiv 0 \text {. }
$$

This, in effect, defines the variable $s$ such that at the ignition position, $r=\hat{r}$, the temperature is given precisely as $T(\hat{r}, t ; \theta) \equiv 1 /(1-s)$. It remains to solve for the function $g(s ; \theta)$ and for $\eta$ at values of $\rho \neq 0$.

The equation satisfied by $\eta$ becomes

$$
\begin{aligned}
1+\frac{\eta_{s}+g^{\prime}}{\theta-g^{\prime}}+\left[\frac{\rho}{2}\left(1-\frac{1}{\alpha+\theta s-g}\right)+\frac{w}{\theta}\right] \eta_{\rho} \\
=\mathscr{H}+\frac{(1-s-\eta / \theta)^{-2}}{\alpha+\theta s-g}\left[\eta_{\rho \rho}+\frac{n \mu}{\hat{r}+\mu \rho} \eta_{\rho}+\frac{2}{\theta(1-s)-\eta} \eta_{\rho}^{2}\right]
\end{aligned}
$$

where

$$
\begin{aligned}
\mathscr{H}= & \frac{\hat{t}-t}{T} \mathscr{R} \\
= & \hat{\Lambda}^{-1}\left[Y_{L}\left(1-\frac{s+\eta / \theta}{\gamma_{L}}\right)\right]^{v_{L}}\left[Y_{R}\left(1-\frac{s+\eta / \theta}{\gamma_{R}}\right)\right]^{v_{R}} \\
& \times\left(1-s-\frac{\eta}{\theta}\right) F\left(\frac{1}{1-s-\eta / \theta}\right) e^{\eta+g}
\end{aligned}
$$


and

$$
w=\left(\frac{\hat{t}-t}{\alpha+\theta s-g}\right)^{1 / 2} v=(\hat{r}+\mu \rho)^{-n} \int_{-\hat{r} / \mu}^{\rho}(\hat{r}+\mu \rho)^{n} \frac{\mathscr{H}}{1-s-\eta / \theta} \partial \rho .
$$

Here, ' denotes ordinary differentiation with respect to $s$, and the quantities $\gamma_{L}$ and $\gamma_{R}$ are defined as $\gamma_{\kappa}=\zeta_{k} Y_{\kappa} /\left(1+\zeta_{\kappa} Y_{\kappa}\right)$, with $\kappa$ representing either $L$ or $R$. Matching with (3.4) requires that $\eta$ behaves as follows:

$$
\begin{aligned}
\eta= & -g-\ln \left(1+\frac{\rho^{2}}{4}\right)-\frac{\ln (\alpha+\theta s-g)}{\alpha+\theta s-g} \frac{5+\hat{n}}{2} \frac{\rho^{2} / 4}{1+\rho^{2} / 4} \\
& +(\alpha+\theta s-g)^{-1}\left[\frac{1+\hat{n}}{2}-\frac{\rho^{2} / 4}{1+\rho^{2} / 4} \ln \left(1+\rho^{2} / 4\right)\right]+O\left[\frac{\ln ^{2}(\alpha+\theta s-g)}{(\alpha+\theta s-g)^{2}}+s\right]
\end{aligned}
$$

provided that $\theta^{-1} \ll s \ll 1$. Applying the condition (4.3), that $\eta \equiv 0$ at $\rho=0$, we require that

$$
g=(\alpha+\theta s)^{-1} \frac{1+\hat{n}}{2}+O\left[\frac{\ln ^{2}(\alpha+\theta s)}{(\alpha+\theta s)^{2}}+s\right]
$$

and

$$
\begin{aligned}
\eta= & -\ln \left(1+\frac{\rho^{2}}{4}\right)-\frac{\ln (\alpha+\theta s)}{\alpha+\theta s} \frac{5+\hat{n}}{2} \frac{\rho^{2} / 4}{1+\rho^{2} / 4} \\
& -(\alpha+\theta s)^{-1} \frac{\rho^{2} / 4}{1+\rho^{2} / 4} \ln \left(1+\rho^{2} / 4\right)+O\left[\frac{\ln ^{2}(\alpha+\theta s)}{(\alpha+\theta s)^{2}}+s\right] .
\end{aligned}
$$

To continue these expansions to order one values of $s$ the following asymptotic forms may be considered for $\eta$ and $g$ :

$$
\begin{aligned}
& \eta=\eta_{0}+\frac{\ln (\alpha+\theta s)}{\alpha+\theta s} \eta_{1}+(\alpha+\theta s)^{-1} \eta_{2}+O\left[\frac{\ln ^{2}(\alpha+\theta s)}{(\alpha+\theta s)^{2}}\right], \\
& g=g_{0}+(\alpha+\theta s)^{-1} g_{1}+O\left[\frac{\ln ^{2}(\alpha+\theta s)}{(\alpha+\theta s)^{2}}\right],
\end{aligned}
$$

with the coefficient functions $\eta_{0}(\rho, s), \eta_{1}(\rho, s)$, etc., being smooth ${ }^{2}$ and continuous functions of $\rho$ and $s$, particularly at $\rho=0$. Together with $g_{0}(s), g_{1}(s)$, etc., they are also independent of $\theta$. The constant $\alpha$ is included in the gauge functions of these expansions so that cases for which $\alpha$ is large may be properly modeled with no extra difficulty, while its omission would lead to unnecessary inaccuracy. As mentioned earlier even cases with $\alpha \gg \theta$, corresponding to very highly supercritical situations, can thus be consistently modeled in an expansion of wide validity.

To first order, equation (4.4) et seq. gives

$$
1+\frac{\rho}{2} \eta_{0_{\rho}}=\left(1-s / \gamma_{L}\right)^{\nu_{L}}\left(1-s / \gamma_{R}\right)^{v_{R}}(1-s) F\left(\frac{1}{1-s}\right) e^{\eta_{0}+g_{0}}
$$

\footnotetext{
${ }^{2}$ It may be thought that the appearance of a singularity in $\eta$ at $\rho=0$ could be associated with a need for an inner matching layer of solution. It is precisely for this reason that solutions in the innermost region must be smooth and nonsingular. Layers for which $r \leqq O(\hat{t}-t)^{1 / 2}$ have already been dismissed [8], [11] as leading to either trivial or nonphysical results. It is shown in [8] that the only other candidate for an innermost layer is $\rho=O(1)$, where $\phi$ and $\eta$ must clearly be smooth and nonsingular.
} 
so that, using condition (4.3) for which $\eta_{0}(0, s)$ must be zero, we have

$$
g_{0}=-\ln \left[\left(1-s / \gamma_{L}\right)^{\nu_{L}}\left(1-s / \gamma_{R}\right)^{v_{R}}(1-s) F\left(\frac{1}{1-s}\right)\right]
$$

and

$$
\eta_{0}=-\ln \left[1+A_{0}^{2}(s) \rho^{2} / 4\right]
$$

Matching with equation (4.9) requires that $A_{0}(0)=1$, but no more can be said about the function of integration $A_{0}(s)$ at this stage. The expansion velocity caused by chemical heating takes the first-order form

$$
\begin{aligned}
w_{0}(\rho, s) & =\frac{\rho^{-\hat{n}}}{1-s} \int_{-\hat{r} \cdot \infty}^{\rho} \rho^{\hat{n}} \frac{\partial \rho}{1+A_{0}^{2} \rho^{2} / 4} \\
& =\frac{1}{(1-s) A_{0}} \cdot \begin{cases}\pi+2 \tan ^{-1}\left(\frac{A_{0} \rho}{2}\right), & \hat{r}>0, \\
2 \tan ^{-1}\left(\frac{A_{0} \rho}{2}\right), & n=0, \quad \hat{r}=0, \\
\frac{2}{A_{0} \rho} \ln \left(1+A_{0}^{2} \rho^{2} / 4\right), & \hat{n}=1, \\
\frac{4}{A_{0}^{2} \rho^{2}}\left[A_{0} \rho-2 \tan ^{-1}\left(\frac{A_{0} \rho}{2}\right)\right], & \hat{n}=2\end{cases}
\end{aligned}
$$

where the product $\hat{r} \cdot \infty$ should be interpreted as representing zero if $\hat{r}=0$ and infinity if $\hat{r}>0$.

At the order $\ln (\alpha+\theta s) /(\alpha+\theta s),(4.4)$ gives

$$
\frac{\rho}{2} \eta_{1_{\rho}}=\frac{\eta_{1}}{1+A_{0}^{2} \rho^{2} / 4}
$$

which solves to give

$$
\eta_{1}=A_{1}(s) \frac{A_{0}^{2} \rho^{2} / 4}{1+a_{0}^{2} \rho^{2} / 4}
$$

with $A_{1}(0)=-(5+\hat{n}) / 2$ for matching. The condition (4.3) is automatically satisfied so that there is no need for an equivalent order in the expansion for $g$.

The order $(\alpha+\theta s)^{-1}$ is more fruitful. The equation for $\eta_{2}$ becomes

$$
\begin{aligned}
\eta_{2}+g_{1} & -\left(1+A_{0}^{2} \rho^{2} / 4\right) \frac{\rho}{2} \eta_{2_{\rho}}-\frac{A_{0}^{2}}{(1-s)^{2}} \frac{1+\hat{n}}{2} \\
& +(\beta+s)\left\{w_{0} A_{0}^{2} \frac{\rho}{2}+\left[\ln \left(1+A_{0}^{2} \rho^{2} / 4\right)-\left(1+A_{0}^{2} \rho^{2} / 4\right)\right] g_{0}^{\prime}\right\} \\
= & {\left[1-2(\beta+s) \frac{A_{0}^{\prime}}{A_{0}}\right] \frac{A_{0}^{4} \rho^{4} / 16}{1+A_{0}^{2} \rho^{2} / 4} } \\
& +\left[1-\frac{A_{0}^{2}}{(1-s)^{2}}-2(\beta+s) \frac{A_{0}^{\prime}}{A_{0}}\right] \frac{A_{0}^{2} \rho^{2} / 4}{1+A_{0}^{2} \rho^{2} / 4}
\end{aligned}
$$

where the constant $\beta=\alpha / \theta$ has been introduced to allow for cases in which $\alpha$ may be large enough for the ratio $\alpha / \theta$ to be of order one or greater. Otherwise $\beta$ may be 
set equal to zero. Applying the condition (4.3) and solving the differential equation, we obtain

$$
g_{1}=\frac{1+\hat{n}}{2} \frac{A_{0}^{2}}{(1-s)^{2}}+(\beta+s) g_{0}^{\prime}
$$

and

$$
\begin{aligned}
\eta_{2}= & \frac{A_{0}^{2} \rho^{2} / 4}{1+A_{0}^{2} \rho^{2} / 4}\left[A_{2}(s)-\left(1-2 \overline{\beta+s} \frac{A_{0}^{\prime}}{A_{0}}\right) \ln \left(1+A_{0}^{2} \rho^{2} / 4\right)\right] \\
& -\overline{\beta+s} g_{0}^{\prime} \ln \left(1+A_{0}^{2} \rho^{2} / 4\right) \\
& -\frac{(\beta+s) /(1-s)}{1+A_{0}^{2} \rho^{2} / 4} \cdot \begin{cases}A_{0} \rho\left[\pi+2 \tan ^{-1}\left(\frac{A_{0} \rho}{2}\right)\right], & \hat{r}>0, \\
A_{0} \rho\left[2 \tan ^{-1}\left(\frac{A_{0} \rho}{2}\right)\right], & n=0, \\
\ln \left(1+A_{0}^{2} \rho^{2} / 4\right), & \hat{r}=0, \\
\frac{4}{3}-\frac{8}{3 A_{0} \rho} \tan ^{-1}\left(\frac{A_{0} \rho}{2}\right), & \hat{n}=1,\end{cases} \\
& +\left\{\frac{A_{0}^{2}}{(1-s)^{2}}-1+2 \overline{\beta+s}\left[\frac{A_{0}^{\prime}}{A_{0}}+\frac{(1+\hat{n})^{-1}}{1-s}\right]\right\} \frac{A_{0}^{2} \rho^{2} / 4}{1+A_{0}^{2} \rho^{2} / 4} \ln \left(\frac{\rho^{2}}{1+A_{0}^{2} \rho^{2} / 4}\right)
\end{aligned}
$$

with $A_{2}(0)=0$ for matching with condition (4.9).

The final term of the latter result potentially has a singular second derivative. Because the expansion for $\eta$ must be smooth at all orders this term has to be excluded, requiring the coefficient of $\ln \rho^{2}$ in equation (4.19) to be set equal to zero. This leads to the equation for $A_{0}$ :

$$
A_{0}^{\prime}+\frac{A_{0} /(1+\hat{n})}{1-s}=A_{0} \frac{1-A_{0}^{2} /(1-s)^{2}}{2(\beta+s)} .
$$

By using the condition for matching, $A_{0}(0)=1$, this equation can be uniquely solved to give

$$
A_{0}^{2}= \begin{cases}\frac{(1-s)^{2},}{(1-s)(\beta+s)} & \hat{n}=0, \\ \frac{(1-s)^{2 / 3}(\beta+s)}{\beta+3\left[(1-s)^{-1 / 3}-1\right]}, & \hat{n}=1, \\ \hat{n}=2 .\end{cases}
$$

Consequently, having applied a smoothness condition to the third order in the expansion for $\eta$, the first order becomes uniquely determined. A similar effect is seen in the derivation [8] of the solution (3.4), which describes the temperature in its early stage of growth.

Unlike the solution (3.4) the solution describing substantial temperature growth does depend on the geometry $\hat{n}$ to first order. This is because the first-order velocity term $w_{0}$ (which is negligible in the early stage) introduces a geometrically dependent element into (4.19), and hence, via the solvability condition (4.20), into the solution (4.21) for $A_{0}$. It should also be noted that the analysis remains valid even for large values of $\beta$, that is for $\alpha \gg \theta$. As mentioned earlier, this corresponds to cases of very highly supercritical thermal runaway, for which conductive and diffusive effects would 
start off being very small indeed and would remain small throughout the evolution. It is reasonable, in such cases, to neglect conduction to first order and arrive at results that are entirely consistent with those presented here. For instance, the behaviour of $A_{0}$ in the limit as $\beta \rightarrow \infty$ agrees precisely with the convective effect, for each value of $\hat{n}$, that thermal expansion would produce in the core of the ignition kernel.

Thus the solution for $T$ can be written as

$$
T=1 /\left\{1-s+\theta^{-1} \ln \left[1+A_{0}^{2} \rho^{2} / 4\right]+O\left[\frac{\ln (\alpha+\theta s)}{\theta(\alpha+\theta s)}\right]\right\}
$$

with

$$
\begin{aligned}
(\hat{t}-t) \hat{\Lambda}\left(1-s / \gamma_{L}\right)^{v_{L}}\left(1-s / \gamma_{R}\right)^{v_{R}}(1-s) F\left(\frac{1}{1-s}\right) \\
=\exp \left\{-\theta s+\theta^{-1}\left[\frac{1+\hat{n}}{2} \frac{A_{0}^{2} /(\beta+s)}{(1-s)^{2}}+v_{L} /\left(\gamma_{L}-s\right)+v_{R} /\left(\gamma_{R}-s\right)\right.\right. \\
\left.\left.+(1-s)^{-1}-(1-s)^{-2} F^{\prime}\left(\frac{1}{1-s}\right) / F\left(\frac{1}{1-s}\right)\right]+O\left[\frac{\ln ^{2}(\alpha+\theta s)}{(\alpha+\theta s)^{2}}\right]\right\}
\end{aligned}
$$

This result fails to behave asymptotically, and therefore becomes invalid as the reaction comes close to completion; the order $\theta^{-1}$ term in the exponent of the right-hand side fails to be small when

$$
s=\gamma_{L}-O\left(\theta^{-1}\right) \quad \text { or } \quad T=1+\zeta_{L} \hat{Y}_{L}-O\left(\theta^{-1}\right)
$$

since $\gamma_{L} \leqq \gamma_{R}$ by definition.

5. Temperature outside the hottest region. As with (3.4), the solution (4.22) successfully describes the growth of the temperature peak, but is only valid in a shrinking neighbourhood of the point $r=\hat{r}$ as $t \rightarrow \hat{t}$. In order to investigate the evolution of the temperature in regions that are left outside this shrinking region at some stage, it is natural to define a family of spatial and time variables $x^{\prime}$ and $\omega^{\prime}$ such that

$$
(r-\hat{r})^{2} \hat{\Lambda}=x^{\prime 2} \frac{4}{A_{0}^{2}\left(\Omega^{\prime}\right)}\left[\theta\left(\beta+\Omega^{\prime}\right)-g\left(\Omega^{\prime}\right)\right] e^{-\theta \Omega^{\prime}+g\left(\Omega^{\prime}\right)}
$$

and

$$
\omega^{\prime}=(t-\hat{t}) \hat{\Lambda} e^{\theta \Omega^{\prime}-g\left(\Omega^{\prime}\right)}
$$

where $\Omega^{\prime}$ is held fixed in the range $0<\Omega^{\prime}<\gamma_{L}$. By using (4.23) to substitute for $s$ and $\rho$ in (4.22), and checking that the result is indeed an asymptotic solution of the governing equations, it is readily found that $T$ is given by

$$
T^{-1}=1-\Omega^{\prime}+\theta^{-1} \ln \left(x^{\prime 2}-\omega^{\prime}\right)+O\left[\frac{\ln \left(\alpha+\theta \Omega^{\prime}\right)}{\theta\left(\alpha+\theta \Omega^{\prime}\right)}\right]
$$

for a given value of $\Omega^{\prime}$.

This result is valid for order one values of $x^{\prime}$ and, in particular, is valid for $x^{\prime}=1$. Therefore it is simpler to write (5.3) in the form

$$
T^{-1}=1-\Omega+\theta^{-1} \ln (1-\omega)+O\left[\frac{\ln (\alpha+\theta \Omega)}{\theta(\alpha+\theta \Omega)}\right]
$$


where $\Omega$ (unlike $\Omega^{\prime}$ ) is not a matter of choice, but is a function of $r$ that satisfies

$$
(r-\hat{r})^{2} \hat{\Lambda}=\frac{4}{A_{0}^{2}(\Omega)}[\theta(\beta+\Omega)-g(\Omega)] e^{-\theta \Omega+g(\Omega)},
$$

and where the corresponding time-like variable grouping $\omega$ is given by

$$
\omega=(t-\hat{t}) \hat{\Lambda} e^{\theta \Omega-g(\Omega)} .
$$

Interestingly, like the solution (3.7), the solution (5.4) becomes singular only as $\omega \rightarrow 1$, or as $t \rightarrow \hat{t}+e^{-\theta \Omega+g(\Omega)} / \hat{\Lambda}$, which is after the ignition time $\hat{t}$. In the absence of deflagration flames this is the time around which the thermal runaway itself would locally drive the reaction toward completion. However, at least in part of the thermal runaway region, it may be anticipated that relatively fast-moving deflagration flames, emerging from the hottest part of the ignition kernel, are likely to destroy the solution before this can happen. The region in which this is the case will be determined later in $\S 7$. In this context (5.4) provides the appropriate "upstream" or "unburnt" temperature (and mixtures strengths from (2.16)), which help to determine the appropriate speed of propagation of any deflagration flames.

6. First completion of the reaction. From (4.22)-(4.24) it can be seen that an appropriate rescaling of space and time to characterize the completion of the reaction in the core of the ignition region is as follows:

$$
t-\hat{t}=\delta \tau \quad \text { and } \quad r-\hat{r}=\hat{\mu} x
$$

where

$$
\begin{gathered}
\delta=\theta^{v} e^{-\theta \gamma_{L}} /\left[\hat{\Lambda} \gamma_{F}^{-v_{F}} \gamma_{X}^{-v_{x}}\left(\gamma_{R}-\gamma_{L}\right)^{v^{\prime}}\left(1-\gamma_{L}\right) F\left(\frac{1}{1-\gamma_{L}}\right)\right], \\
\hat{\mu}^{2}=\delta \theta \frac{\beta+\gamma_{L}}{\hat{A}_{0}^{2} / 4}
\end{gathered}
$$

The constant $\hat{A}_{0}$ is written for $A_{0}\left(\gamma_{L}\right)$ and the constants $v$ and $v^{\prime}$ are defined such that

$$
\left(v, v^{\prime}\right)= \begin{cases}\left(v_{L}, v_{R}\right), & \gamma_{R}-\gamma_{L} \gg \theta^{-1}, \\ \left(v_{L}+v_{R}, 0\right), & \gamma_{R}-\gamma_{L}=O\left(\theta^{-1}\right) .\end{cases}
$$

It is also appropriate to define the variable $y$ to characterize the approach of temperature to its maximum value,

$$
y=\theta\left[\gamma_{L}-(1-1 / T)\right]
$$

The equation satisfied by $y$ then becomes

$$
y_{\tau}+\frac{v}{\theta} y_{x}+\mathscr{G}=\frac{\hat{A}_{0}^{2} /\left(1-\gamma_{L}+y / \theta\right)^{2}}{4\left(\beta+\gamma_{L}\right) \theta}\left[y_{x x}+\frac{n \hat{\mu}}{\hat{r}+\hat{\mu} x} y_{x}-\frac{2 / \theta}{1-\gamma_{L}+y / \theta} y_{x}^{2}\right]
$$

where

$$
\begin{gathered}
\mathscr{G}=\frac{(\sigma+y)^{\nu_{R}}}{\sigma^{v^{\prime}}} y^{\nu_{L}} e^{-y}\left(1+\frac{y / \theta}{1-\gamma_{L}}\right) F\left(\frac{1}{1-\gamma_{L}+y / \theta}\right) / F\left(\frac{1}{1-\gamma_{L}}\right), \\
v=(\hat{r}+\hat{\mu} x)^{-n} \int_{-\hat{r} / \hat{\mu}}^{x}(\hat{r}+\hat{\mu} x)^{n} \frac{\mathscr{G}}{1-\gamma_{L}+y / \theta} \partial x
\end{gathered}
$$

and

$$
\sigma=\theta\left(\gamma_{R}-\gamma_{L}\right)
$$


Expanding the solution for $y$ in the form

$$
y=y_{0}(x, \tau)+O\left(\theta^{-1}\right)
$$

leads to the equation for $y_{0}$,

$$
y_{0_{\tau}}=- \begin{cases}y_{0}^{\nu_{L}} e^{-y_{0}}, & \sigma \gg 1, \\ \left(\sigma+y_{0}\right)^{v_{R}} y_{0}^{\nu_{L}} e^{-y_{0},}, & \sigma=O(1) .\end{cases}
$$

The case with $\sigma=O(1)$ represents a near stoichiometric mixture of fuel and oxidant. Equation (6.10) can be solved for time $\tau$ in terms of $y_{0}$ :

$$
\begin{aligned}
& \tau=f_{0}(x)-\Psi\left(y_{0} ; \hat{y}_{0}\right), \quad \text { with } \\
& \Psi\left(y_{0} ; \hat{y}_{0}\right)= \begin{cases}\int_{\hat{y}_{0}}^{y_{0}} z^{-v_{L}} e^{z} d z, & \sigma \gg 1, \\
\int_{\hat{y}_{0}}^{y_{0}}(\sigma+z)^{-v_{R}} z^{-v_{L}} e^{z} d z, & \sigma=O(1),\end{cases}
\end{aligned}
$$

where $\hat{y}_{0}$ is a constant and $f_{0}(x)$ is a function of integration.

It is necessary to match this solution with the results $(4.22)$ and (4.23) if $f_{0}(x)$ is to be determined. Writing s for $\gamma_{L}-s$, we can see from (4.22) and (4.23) that, for $1 \gg \diamond \gg \theta^{-1}$,

$$
\tau=-\frac{e^{\theta_{\diamond}}}{(\theta\lrcorner)^{v}}\left[1+\frac{v}{\theta\lrcorner}+o(\theta s)^{-1}\right]
$$

and

$$
y=\theta s+\ln \left(1+\hat{A}_{0}^{2} \rho^{2} / 4\right)+O\left(\frac{\ln \left(\alpha+\theta \gamma_{L}\right)}{\alpha+\theta \gamma_{L}}\right)
$$

with

$$
\frac{\hat{A}_{0}^{2} \rho^{2}}{4}=\frac{x^{2}}{-\tau} \frac{\beta+\gamma_{L}}{\beta+\gamma_{L}-\jmath-g / \theta} .
$$

This matches automatically with (6.11) for large values of $y$ and fixed $x$, without yielding any information about $f_{0}(x)$. In order to obtain this information it is necessary to match spatial derivatives of $y$. This requires that

$$
y_{0}^{v} e^{-y_{0}}\left[1+O\left(y_{0}^{-1}\right)\right] f_{0}^{\prime}=2 x\left(\theta_{\lrcorner}\right)^{\nu} e^{-\theta_{\diamond}} /\left[1+x^{2}\left(\theta_{\lrcorner}\right)^{\nu} e^{-\theta_{\lrcorner}}\right],
$$

which can only be satisfied if $f_{0}^{\prime}(x)=2 x$. When the constant of integration is absorbed into the definition of $\hat{y}_{0}$ this condition integrates to give

$$
x^{2}-\tau=\Psi\left(y_{0} ; \hat{y}_{0}\right)
$$

in which the constant $\hat{y}_{0}$ still remains unspecified by the matching process.

In fact, this should not be surprising since $\hat{r}$ and $\hat{t}$ themselves have been specified no more precisely than (2.23), which still leaves room for the addition of an arbitrary constant to the final definition of $\tau$. A reasonable definition, to fix the solutions, would be to specify $\hat{r}$ and $\hat{t}$ as (say) the position and time at which the reaction first reaches a local maximum value. From (6.10) it can be seen that the appropriate choice of $\hat{y}_{0}$ for this is such that

$$
v / \hat{y}_{0}+v^{\prime} /\left(\sigma+\hat{y}_{0}\right)=1
$$


An interesting feature of the solution (6.16) is its quadratic wavelike behaviour, with temperature being a function of $x^{2}-\tau$. Returning to the scalings (6.1) and (6.4), we have that $T$ satisfies

$$
t-\hat{t}-\frac{\hat{A}_{0}^{2} / 4}{\beta+\gamma_{L}} \frac{(r-\hat{r})^{2}}{\theta} \sim-\delta \Psi\left\{\theta\left[\gamma_{L}-(1-1 / T)\right] ; \hat{y}_{0}\right\}
$$

For small enough values of $r-\hat{r}$, the path along which $\theta\left[\gamma_{L}-(1-1 / T)\right]$ takes a particular order one value (for example, $\hat{y}_{0}$ ) is therefore asymptotically close to the path

$$
t=\hat{t}+\frac{\hat{A}_{0}^{2} / 4}{\beta+\gamma_{L}} \frac{(r-\hat{r})^{2}}{\theta} .
$$

For $\Omega$ close to $\gamma_{L}$, this corresponds exactly to the path at which the solution (5.4) becomes singular (as $\omega \rightarrow 1$ ) so that (6.18) matches neatly with the latter result.

Physically, (6.19) represents a parabolic flame path, the movement of which is controlled by spatial variation in the thermal runaway process which, at this stage, is the only significant mechanism responsible for driving the reaction to completion. Although conduction and diffusion are relevant in determining the overall structure of the thermal runaway, their immediate effect in (6.5) is negligible. They do not play a direct role in guiding this early "thermal-runaway flame," which can thus be clearly distinguished from a deflagration flame.

Some solutions for $y_{0}$ in terms of $x$ and $\tau$ are shown in Fig. 1 for various values of $v_{L}$ with $\sigma \gg 1$. In all cases, the wavelike behaviour of the temperature can be seen to emerge as a moving front of temperature increase. As it is driven outward, the region

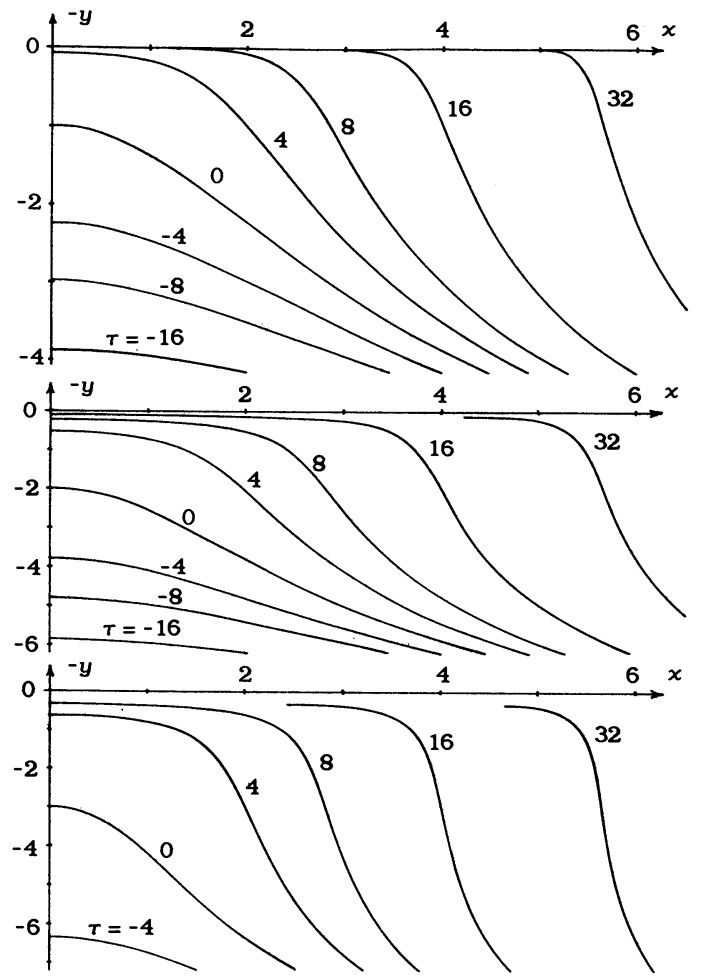

FIG. 1. Temperature profiles in the final-stage of thermal runaway with $\sigma \gg 1$ and with (a) $v_{L}=1,(\mathrm{~b})$ $v_{L}=2$, and $(\mathrm{c}) v_{L}=3$. 
of sharpest temperature rise also becomes narrower and, correspondingly, the region where the reaction is strongest becomes smaller. Indeed, differentiation of $(6.16)$ shows that, as it moves outward close to the path $x=X(\tau)=\sqrt{\tau}$, the thickness of the reacting region decreases like $1 /|X|$, and, $y_{x x}$ increases in proportion to $X^{2}$. The analysis fails when the diffusion terms of (6.5) become significant, that is, in the region

$$
|x|=\sqrt{\tau}+O\left[\theta\left(\beta+\gamma_{L}\right)\right]^{-1 / 2}: \quad \tau=O\left[\theta\left(\beta+\gamma_{L}\right)\right] .
$$

When we use (6.7) and (6.16) the velocity component $v$ can be evaluated to first order in the form

$$
\begin{aligned}
v_{0} & =x^{-\hat{n}} \int_{-\hat{r} \cdot \infty}^{x} x^{\hat{n}} \frac{y_{0_{\tau}}}{1-\gamma_{L}} \partial x \\
& =v_{0}(0, \tau)+\frac{x^{-\hat{n}}}{2\left(1-\gamma_{L}\right)} \int_{y_{0}(x, \tau)}^{y_{0}(0, \tau)} x^{\hat{n}-1} \partial y_{0},
\end{aligned}
$$

the integrals being performed at constant time. Some velocity profiles are shown in Fig. 2. It can be seen that velocities increase as the fastest reaction rates are approached, but as the reaction region moves outward and becomes narrower, the maximum velocity decreases again as shown in Fig. 3. Thus within the time-region $\tau=O(1)$, the nondimensional velocity $u=T \partial T / \partial r+v / \theta$ reaches an overall maximum value such that

$$
u_{\max }^{2}=O\left[\left(\beta+\gamma_{L}\right) e^{\theta \gamma_{L}} / \theta^{1+\nu}\right] .
$$

It must be remembered from $\S 2$ that gas velocities have been assumed to remain small enough, compared with the typical sound speed $a_{c}^{*}$, for pressure gradients to

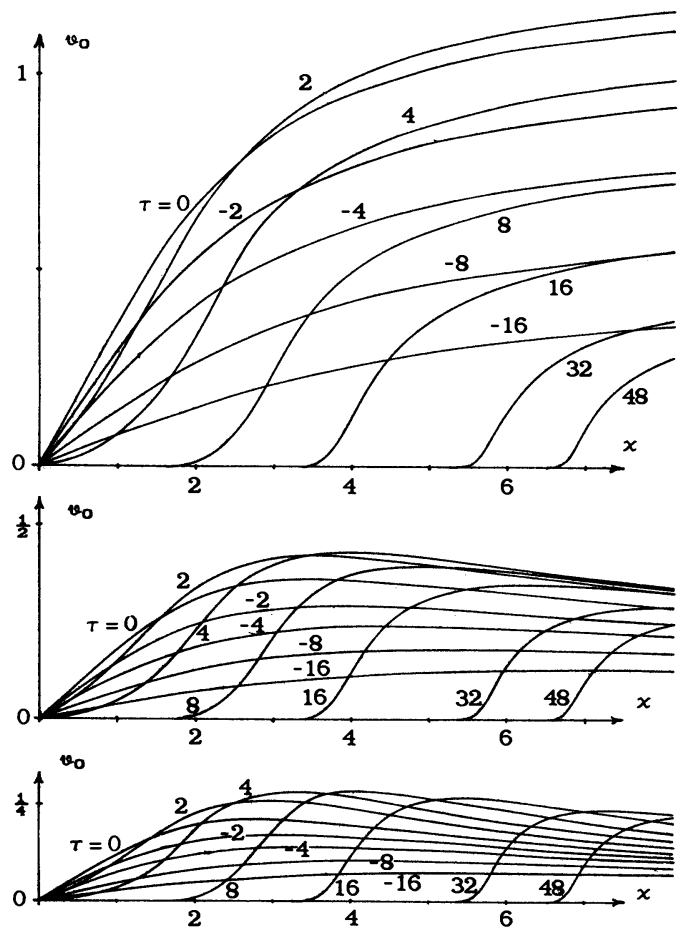

FIG. 2. Velocity profiles in the final stage of thermal runaway with $\sigma \gg 1, v_{L}=1, \hat{r}=0$ and (a) $n=0$, (b) $n=1$, and (c) $n=2$. 


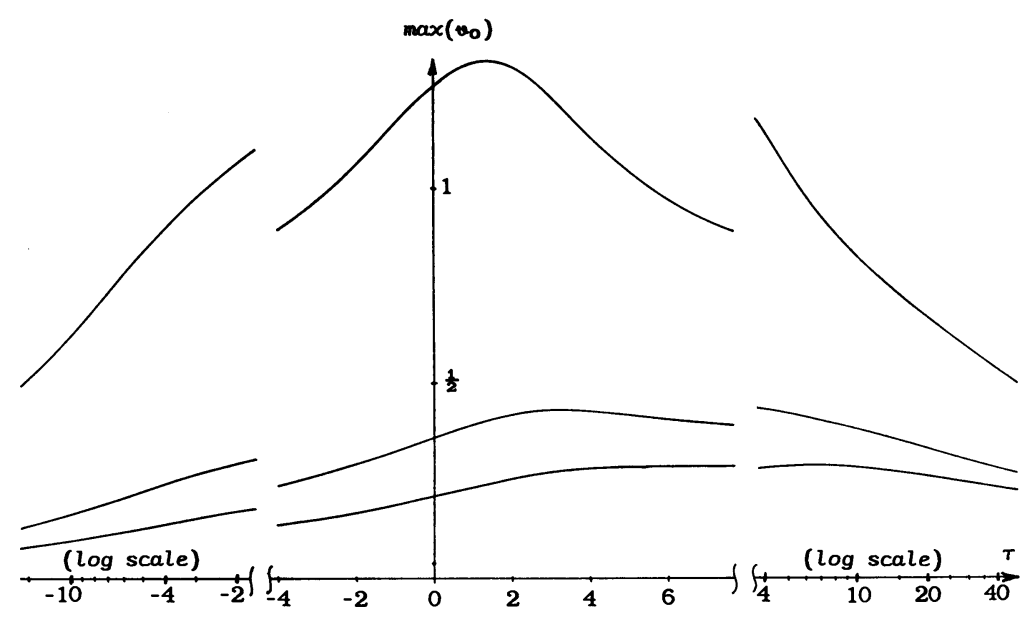

FIG. 3. Variation of the maximum velocity with time for cases having $\sigma \gg 1, v_{L}=1, \hat{r}=0$ and (a) $n=0$, (b) $n=1$, and (c) $n=2$.

be negligible in the model. If the maximum dimensional velocity were to become as large as the order of $\theta^{-1}$ times the sound speed, the model would then break down and significant dynamic pressure/velocity interactions would have to be considered. Changes that would occur in the nature of the solution have been described elsewhere [12].

This transition arises essentially when acoustic and induction times, in the heart of the ignition region, become of the same order. A final-stage induction time (which estimates the time remaining to complete combustion) can be identified as

$$
\hat{t}_{c}^{\#}=\theta^{v} e^{-\theta \gamma_{L}} t_{c}^{\#}
$$

where $t_{c}^{\#}$ is the initial induction time identified in (2.2). Correspondingly a final-stage acoustic time, which measures the time taken for acoustic waves to cross the core of the ignition region, is given by

$$
\begin{aligned}
\hat{t}_{a}^{\# 2} & =\left(\beta+\gamma_{L}\right) \theta^{1+v} e^{-\theta \gamma_{L} k_{c}^{\#} t_{c}^{\#} / a_{c}^{\# 2}} \\
& =\left(\beta+\gamma_{L}\right) \theta k_{c}^{\#} \hat{t}_{c}^{\#} / a_{c}^{\# 2} .
\end{aligned}
$$

As shown in [12], dynamic pressure interactions can be neglected provided the acoustic time remains short compared with the reaction time. If this is the case then thermal expansion effects have time to propagate outward (leading to the expansion velocity $v / \theta)$, and pressure increases remain fairly small. Otherwise thermal expansion simply does not have time to establish itself, and density changes become small while the absolute pressure builds up significantly. Thus, for the model that has been used here to remain valid, it is necessary that $\hat{t}_{a}^{\#}$ should remain small compared with $\hat{t}_{c}^{\#}$. When we use the expressions above, this amounts to the requirement

$$
\hat{t}_{c}^{\#} \gg\left(\beta+\gamma_{L}\right) \theta k_{c}^{\#} / a_{c}^{\# 2} \text {. }
$$

We may recall that $k_{c}^{\#} / a_{c}^{\# 2}$ is a measure of the molecular collision time. Thus $\widehat{\mathrm{Ka}}=\hat{t}_{c}^{\#} a_{c}^{\# 2} / k_{c}^{\#}$ represents a typical value of the von Kármán number (defined in $\S 2$ ) during the final stage of the thermal runaway when the reaction rate will be fastest, and thus the von Kármán number will be close to its minimum value. For physical reasons, $\widehat{\text { Ká }}$ must nevertheless still be a large number. The inequality (6.25) can thus 
be written as

$$
\widehat{\mathrm{Ká}} \gg \alpha+\theta \gamma_{L}
$$

requiring that the minimum value of the von Kármán number must, nevertheless, be large compared with $\left(\beta+\gamma_{L}\right) \theta$. Recalling that $\theta$ is large, it is conceivable for this inequality to be violated (resulting in more significant pressure changes) for realistic chemical reactions; large values of $\alpha$ could produce the same effect. In other words, a transition toward constant density behaviour can be predicted for values of $\alpha+\theta \gamma_{L}$ greater than, or of the order of $\widehat{\mathrm{Ka}} / \theta$.

7. Emergence of deflagration-flames. Without solving for the detailed structure of the transition process, it is possible to predict the emergence of deflagration flames quite simply on phenomenological lines. The speed of the thermal-runaway flame, traveling along the path (6.19), is found by differentiation to be

$$
\left.\frac{\partial r}{\partial t}\right|_{T} \sim \frac{2 \theta\left(\beta+\gamma_{L}\right)}{\hat{A}_{0}^{2}} /(r-\hat{r})=\frac{2 \theta\left(\beta+\gamma_{L}\right)}{\hat{\mu} \hat{A}_{0}^{2}} / x .
$$

Although initially infinite (at $r=\hat{r}$ ), the speed of this flame decreases as the reciprocal of the distance traveled. It must eventually become less than the speed with which a deflagration-flame would propagate under the same locally prevailing conditions. At this stage deflagration, rather than the thermal-runaway process, will begin to dominate the remaining combustion. If ignition is considered to be the process by which self-sustaining combustion waves, such as deflagration flames, are initiated, then ignition could be said to be complete.

With a burnt temperature of $T_{b}=1+\zeta_{L} \hat{Y}_{L}$ and an unburnt temperature of

$$
T_{u}=T_{b}-\theta^{-1} T_{b}^{-2}\left[\ln x^{2}+v \ln \left(\ln x^{2}\right)\right]+O\left(\theta^{-1}\right)
$$

(from (5.4) for $\omega<1$ ) the deflagration flame-speed is shown in the Appendix to be

$$
S_{d}=\theta^{-1 / 2} \hat{\mu}^{-1}\left(2 / \hat{A}_{0}\right)\left(\beta+\gamma_{L}\right)^{1 / 2} T_{b}^{3} C_{0} /\left(T_{b}-T_{u}\right) .
$$

The two flame speeds become comparable and so a transition, which allows a deflagration flame to propagate ahead, will take place when the flame passes through the region

$$
x \approx\left[\theta\left(\beta+\gamma_{L}\right)\right]^{1 / 2} \ln \left[\theta\left(\beta+\gamma_{L}\right)\right] /\left(T_{b}^{5} C_{0} \hat{A}_{0}\right) .
$$

Note that this region is greater by a factor of order $\ln \left[\theta\left(\beta+\gamma_{L}\right)\right]$ than the region (6.20) in which the reaction zone of the deflagration flame comes into existence. A sketch of the situation is shown in Fig. 4.

Subsequent combustion will be dominated by the propagation of deflagration flames and the possible emergence of diffusion flames as described by Liñan and Crespo [18] and Dold and Clarke [13]. The appropriate premixed flame-speed for this is derived in the Appendix.

8. Conclusions. The effect of heat conduction on the progress of thermal runaway has been analysed and asymptotic solutions have been presented to describe temperature growth from an early stage to the time when premixed flames begin to emerge from the core of an ignition kernel. Provided gasdynamic pressure effects remain small, the results are valid from marginally supercritical to very highly supercritical cases where conductive effects may be small right from the start. Conditions have been identified under which substantial pressure increases could be produced (as described in [12]). These depend on the early effect of conduction being small enough, or the ignition kernel being large enough at some stage for reaction and acoustic times to 


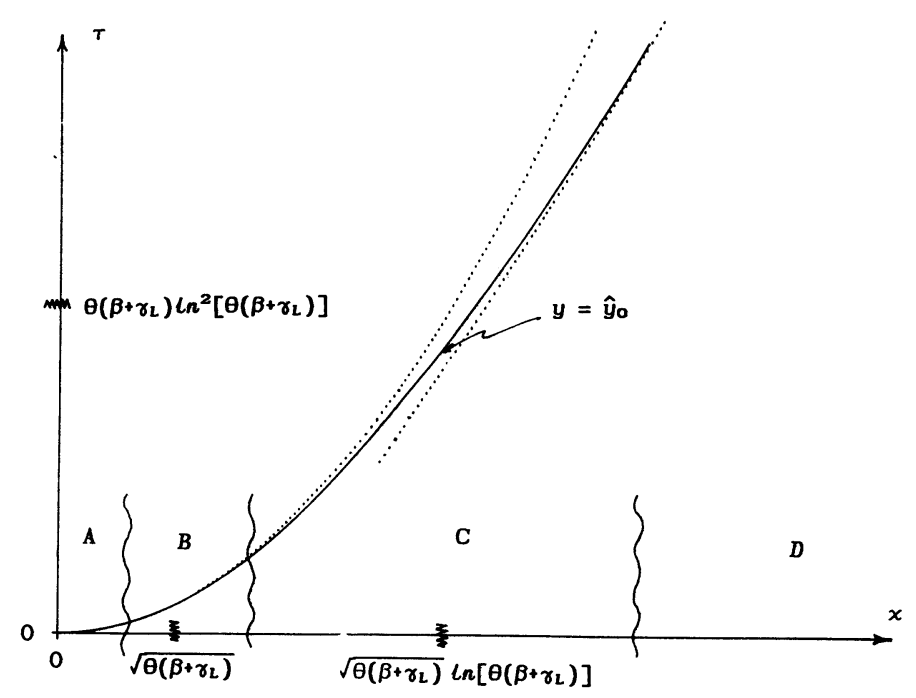

FIG. 4. Sketch of the progress from a thermal-runaway flame to a quasi-steady deflagration flame through the regions in which:
A. conductive effects are negligible;
B. conductive effects become significant in a reaction-zone;
C. transition occurs to a deflagration flame structure; and
D. quasi-steady deflagration flames propagate ahead.

become similar in magnitude. The progressive diminishing of the relative effect of conduction, as thermal runaway proceeds, contributes to the likelihood of this situation arising.

Appendix. Deflagration flame speed. It is sufficient here to analyse the propagation of premixed flames away from the centre, $r=0$. A simple extension taking into account convection associated with the thermal expansion caused by any inward propagating flame [13] could be used to extend the results to cases where $\hat{r} \neq 0$. The equation

$$
\begin{aligned}
T_{t}+\frac{v}{\theta} T_{r}= & T^{2}\left(T_{r r}+\frac{n}{r} T_{r}\right)+\theta^{-1}\left[Y_{L}\left(1-\frac{1-1 / T}{\gamma_{L}}\right)\right]^{\nu_{L}} \\
& \times\left[Y_{R}\left(1-\frac{1-1 / T}{\gamma_{R}}\right)\right]^{\nu_{R}} T F(T) e^{\theta(1-1 / T)},
\end{aligned}
$$

must be satisfied, with $T \sim T_{u}(r, t ; \theta)$ ahead of any flame, and $T \sim T_{b}(r, t ; \theta)$ behind a flame (recalling that $\left.T_{b}=1+\zeta_{L} Y_{L}=\left(1-\gamma_{L}\right)^{-1}\right)$. As Zeldovich [19] has pointed out, a quasi-steady analysis can be used to derive a premixed flame speed, with $T_{u}$ and $T_{b}$ taken to be locally constant, provided that $T_{u}$ and $T_{b}$ vary slowly on the length scale of a preheat zone thickness. In the region of the ignition kernel where deflagration flames begin to emerge $((7.4))$ this condition is satisfied. Also the geometrically dependent term $(n / r) T_{r}$ in (A1) is negligible so that the flame may be considered as quasi-one-dimensional.

Because of this, the passage of a deflagration flame can be modeled in preheat and reaction zones using

$$
T \sim T_{p}(z) \text { and } T \sim T_{b}-\theta^{-1} T_{b}^{2} T_{f}(\theta z),
$$

respectively, where $z=k r-k^{2} V t$ with

$$
k^{2}=\left(Y_{L} / \gamma_{L}\right)^{v_{L}}\left(Y_{R} / \gamma_{R}\right)^{v_{R}}\left(\gamma_{R}-\gamma_{R}\right)^{v^{\prime}} T_{b}^{-3} F\left(T_{b}\right) \theta^{-2-v} e^{\theta \gamma_{L}} .
$$


The parameters $v, v^{\prime}$, and $\sigma$ are defined in (6.3) and (6.8). Analysis of the reaction zone gives $T_{f}^{\prime}$ in terms of $T_{f}$ as follows:

$$
T_{f}^{\prime 2}= \begin{cases}2 \int_{0}^{T_{f}} y^{\nu_{L}} e^{-y} d y, & \sigma \neq O(1), \\ 2 \int_{0}^{T_{f}} y^{\nu_{L}}(\sigma+y)^{\nu_{R}} e^{-y} d y, & \sigma=O(1),\end{cases}
$$

which shows $T_{f}^{\prime 2}$ approaching an order one constant value, $C_{0}^{2}\left(v_{L}, v_{R}, \sigma\right)$, given by the finite limit of the right-hand sides of (A4) as $T_{f} \rightarrow \infty$. For example, with $\sigma \gg 1$, this gives $C_{0}=\sqrt{2 \Gamma\left(v_{L}+1\right)}$. Analysis of the preheat zone leads to the result for $z$ in terms of $T_{p}$

$$
\bar{V} z=T_{u}^{2} \ln \left[\left(T_{b}-T_{u}\right) /\left(T_{p}-T_{u}\right)\right]+T_{u}\left(T_{b}-T_{p}\right)
$$

where $\bar{V}=V-k^{-1} v / \theta$. Since the reaction is only significant in the reaction zone, $\bar{V}$ is constant in the preheat zone, and indeed $v$ can be evaluated to give

$$
k^{-1} v / \theta \sim T_{b}^{3} T_{f}^{\prime},
$$

which tends to the constant value $T_{b}^{3} C_{0}$ in the preheat zone.

We have that matching of the temperature gradient between the two regions leads to the following expression for $V$ :

$$
V=T_{b}^{4} C_{0} /\left(T_{b}-T_{u}\right) .
$$

Note that, had $k^{-1} v / \theta$ been zero (as it would be for a flame propagating toward $r=0$, with still gases ahead of it) this result would have been reduced by the factor $T_{b} / T_{u}$, which is exactly the amount of volumetric expansion caused through the heating of the gases by the flame. The outwardly propagating flame can thus be seen to have a flames begin to emerge $((7.4))$ this condition is satisfied. Also the geometrically dependent term $(n / r) T_{r}$ in (A1) is negligible so that the flame may be considered as quasi-one-dimensional.

$$
\left.\frac{\partial r}{\partial t}\right|_{z}=S_{d}=\left[\left(Y_{L} / \gamma_{L}\right)^{v_{L}}\left(Y_{R} / \gamma_{R}\right)^{v_{R}}\left(\gamma_{R}-\gamma_{L}\right)^{v^{\prime}} T_{b}^{5} F\left(T_{b}\right)\right]^{1 / 2} C_{0} \frac{\theta^{-1-v / 2} e^{\theta \gamma_{L} / 2}}{T_{b}-T_{u}}
$$

\section{REFERENCES}

[1] D. A. Frank-KAMEnetskil, On the mathematical theory of thermal explosions, Acta Physiocochimica U.R.S.S., 16 (1942), p. 357.

[2] - Diffusion and Heat Exchange in Chemical Kinetics, Princeton University Press, Princeton, NJ, 1955.

[3] Ya. B. Zeldovich, G. I. Barenblatt, V. B. Librovich, and G. M. Makhviladze, The time-independent theory of thermal explosions, in The Mathematical Theory of Combustion and Explosions, Plenum Press, New York, 1985, pp. 143-185.

[4] J. W. DOLD, On the autoignition and combustion of a finite region of gaseous fuel, Ph.D. thesis, Cranfield Institute of Technology, Bedfordshire, U.K., 1979.

[5] A. K. KAPILA, Reactive-diffusive system with Arrhenius kinetics: dynamics of ignition, SIAM J. Appl. Math., 39 (1980), pp. 21-36.

[6] D. R. KASSOY AND J. POLAND, The thermal explosion confined by a constant temperature boundary: I-the induction period solution, SIAM J. Appl. Math., 39 (1980), pp. 412-430.

[7] - The thermal explosion confined by a constant temperature boundary: II-the extremely rapid transient, SIAM J. Appl.. Math., 41 (1981), p. 231. 
[8] J. W. Dold, Analysis of the early stage of thermal runaway, Quart. J. Mech. Appl. Math., 38 (1985), pp. 361-387.

[9] A. A. LIÑÁN AND G. JOULIN , private communication, 1984.

[10] J. W. DOLD AND J. F. CLARKE, Ignition and criticality of a finite quantity of gas released in the atmosphere, in Proc. 20th International Symposium on Combustion, Michigan, 1984, pp. 1861-1867.

[11] J. BEBERNES AND D. R. KASSOY, Characterising self-similar blow-up, in Mathematical Modelling of Combustion and Related Topics, C-M. Brauner and C. Schmidt-Lainé, eds., Martinus Nijhoff, Dordrecht, 1988, pp. 383-392.

[12] J. W. DoLD, Dynamic transition of a self-igniting region, in Mathematical Modelling of Combustion and Related Topics, C-M. Brauner and C. Schmidt-Lainé, eds., Martinus Nijhoff, Dordrecht, 1988, pp. 461-470.

[13] J. W. Dold AND J. F. ClaRKe, in Combustion of a finite quantity of gas released in the atmosphere, in Proc. 21st International Symposium on Combustion, Munich, Federal Republic of Germany, 1986, pp. 1349-1356.

[14] F. A. Williams, Combustion Theory, 2nd ed., Benjamin/Cummings, CA, 1985.

[15] D. R. KASSOY, The supercritical spatially homogeneous thermal explosion-initiation to completion, Quart. J. Mech. Appl. Math., 30 (1977), pp. 71-89.

[16] J. D. Buckmaster, An Introduction to Combustion Theory, in The Mathematics of Combustion, J. D. Buckmaster, ed., Frontiers 2, Society for Industrial and Applied Mathematics, Philadelphia, PA, 1985, p. 14.

[17] J. F. Clarke And M. MCChesney, Dynamics of Relaxing Gases, Butterworth, London, 1976, pp. 54-58.

[18] A. A. LiñÁN AND A. CRESPO, An asymptotic analysis of unsteady diffusion flames for large activation energy, Comb. Sci. Tech., 14 (1976), pp. 95-117.

[19] Y. ZELDOVICH, Flame propagation in a substance reacting at initial temperature, Combustion and Flame, 39 (1980), pp. 219-224.

[20] T. vON KÁRMÁN, Models in thermogasdynamics, in I Modelli Nella Technica, Atti del Convegno di Venezia, 1 (1955), pp. 643-651. 